\title{
A Roadmap for Delivering the Promise of Gaia
}

\author{
T. Prusti ${ }^{1}$, C. Aerts ${ }^{2}$, E. K. Grebel ${ }^{3}$, C. Jordi ${ }^{4}$, S. A. Klioner ${ }^{5}$, \\ L. Lindegren ${ }^{6}$, F. Mignard ${ }^{7}$, S. Randich ${ }^{8}$ and N. A. Walton ${ }^{9}$ \\ ${ }^{1}$ ESA, ESTEC, The Netherlands \\ email: tprusti@rssd.esa.int \\ ${ }^{2}$ Katholieke Universiteit Leuven, Faculteit Wetenschappen, Belgium \\ ${ }^{3}$ Universität Heidelberg, Zentrum für Astronomie, Germany \\ ${ }^{4}$ Universitat de Barcelona, Facultat de Fisica, Spain \\ ${ }^{5}$ Technische Universität Dresden, Germany \\ ${ }^{6}$ Lund Observatory, Sweden \\ ${ }^{7}$ Observatoire de la Côte d'Azur, France \\ ${ }^{8} \mathrm{INAF}$, Osservatorio Astrofisico di Arcetri, Italy \\ ${ }^{9}$ University of Cambridge, Institute of Astronomy, United Kingdom
}

\begin{abstract}
Gaia development is in full speed aiming to the launch in December 2011. While the entities formally responsible to make Gaia happen are very focused to be ready in time, it is necessary to explore the readiness of the wider scientific community to exploit Gaia data in the future. The GST sees a role for itself in building and enabling the community to use the Gaia catalogues when they become available. This paper gives the background for the current activities the GST is taking to promote the community to start preparations for the exploitation of Gaia data.
\end{abstract}

Keywords. space vehicles, surveys, astrometry

\section{Introduction}

Gaia is an ESA space astrometry mission with main goals related to the origin, structure and evolutionary history of our Galaxy. The scientific questions are addressed by Gaia with an all sky survey covering in addition to astrometry also photometry and spectroscopy. The astrometric and photometric survey will be down to the 20th magnitude leading to measurements of about one billion stars. The spectroscopic instrument is less sensitive providing an all sky survey down to the 17th magnitude. Due to the all sky aspect the scientific results from Gaia will reach beyond those of the primary goals. Gaia will contribute in many areas of astronomy, stellar astrophysics, solar system studies and general relativity just to mention a few. The details of the scientific performance and applications to the topic of this meeting are covered by the accompanying Gaia presentation by Bailer-Jones (2008) in these proceedings.

This contribution will address, as the title suggests, the way the scientific results will be optimally achieved. The roadmap to deliver Gaia has two elements. The functional part of building the spacecraft and data processing system to provide the results are essential pre-conditions to make Gaia a success. The status and organisational structure for this are briefly summarised in this contribution. The main emphasis of this paper is on the less direct organisational aspect: how to involve the "astronomical world" outside Gaia to maximise the scientific output from the mission. 


\section{Overview of the Gaia project}

At the top level the Gaia project can be divided into ESA, industrial and scientific community elements. In the case of Gaia the whole spacecraft including the payload is built by industry with EADS Astrium as the prime industrial contractor. Another industrial component in the mission is the launcher which is the Soyuz/Fregat managed by Starsem. The launch will take place in Kourou. The ESA role is the traditional management of the industrial contracts and participation in the operations. The community has a crucial role in Gaia by providing the Data Processing and Analysis Consortium (DPAC) which is responsible of producing the immediate scientific output of the mission: the Gaia catalogue. In order to ensure the optimal scientific operations, the ESA Science Operations Centre has been integrated into DPAC to form a single entity responsible of the scientific operations and data processing.

All the above mentioned mission elements have been selected and are in full speed to complete their part of the project. EADS Astrium passed successfully the Preliminary Design Review (PDR) 2007 and is currently in Phase C/D completing the details of the design and building the flight hardware. The Data Processing and Analysis Consortium was formally approved 2007 and the same year passed successfully the System Requirements Review (SRR) together with the ESA Mission Operations Centre (MOC). Currently Gaia is on schedule for a launch in December 2011.

In addition to the entities responsible of implementing Gaia, the project, like every ESA project, has also advisory bodies providing guidance and recommendations to all aspects of the missions. For Gaia the closest advisory body is the Gaia Science Team (GST), who are authoring this roadmap paper, with the responsibility to provide the scientific advise for all aspects of the project.

\section{Gaia project and the astronomical community}

Although the Gaia project elements already in place form a complete entity that will make Gaia happen, the scientific part of Gaia cannot be separated from the overall scientific environment. The astronomers in DPAC are part of the overall scientific community, but in addition Gaia needs the scientific world outside the strict boundaries of the project. By definition this interface cannot be formalised, but neither can it be left on its own with the hope that the matter sorts out by itself as a natural process between scientists.

The classical example of (difficult) project interaction with the rest of the astronomical world is the need of calibration observations. Calibration observations for another project is not exactly the favourite proposal for any time allocation committee. In Gaia the ground based observations needs are coordinated by the DPAC entity GBOG (Ground Based Observations for Gaia). GBOG is facing the typical problem of calibration proposal writing where the proposal needs to be scientifically motivated rather than by Gaia calibration needs. While many calibration observations can be obtained in observing projects with a direct scientific goal next to the Gaia calibration needs, there are always calibration observations needed which simply cannot be embedded in a regular observing proposal. Yet these observations are needed for the optimal science from Gaia. This problem is generic and by no means unique to Gaia. In this topic the attempt to coordinate ground based facilities in Europe under the ASTRONET consortium is an interesting development and it is desirable that any concept coming out from that process will also consider the need for calibration observations.

When moving away from the interface between Gaia and ground based calibration observations, the needs become less clear. In principle the baseline is very clear. Gaia is 
based on data release policy without any proprietary rights. This means that Gaia needs to document the catalogue and its contents when it is published, and the work after that is up to the scientific community. Undoubtedly this way the scientific harvest will also follow, but it is equally sure that with good preparations the exploitation is not only quicker, but also with more depth and breadth. This is an area where the GST has the role to bridge the gap between Gaia and the outside world for maximum scientific return.

In addition to the final Gaia catalogue, intermediate catalogues and science alerts will be published in the course of the mission. It is obvious that any scientific follow up based on the Gaia results is left to the community at large. The issue is that some follow up observations may be time consuming to obtain. In this kind of cases it is better not to wait till the publication of the Gaia catalogue, but rather to inform the community to start "follow up" preparations already earlier. Another example of early preparation need is e.g. a requirement to follow up with facilities which are available now but won't be available in the Gaia era. A pre-requisite of this activity is to provide the community with information of Gaia performances, including biases or shortcomings, and schedule also in the context of other contemporary survey projects. Rather than assuming the step of informing the community to be a task to be completed as soon as possible, we should be looking into an iterative and interactive process. A positive side effect of conducting this process interactively is the community building aspect. We must not only worry about having all the follow up preparations done in time, but also ensure a wide enough community to exploit the data. The GST is going to be actively involved in this process.

As an example of a concrete action taken at the time when these proceedings are written, the GST is currently (September 2008) soliciting expressions of interest for the GREAT programme (Gaia Research for European Astronomy Training; see details in http://www.ast.cam.ac.uk/GREAT/). The aim is to build a research network for the promotion of topical workshops, conferences, training events, exchange visits, publications and outreach activities addressing the major scientific issues that the Gaia satellite will impact upon.

\section{Conclusions}

Gaia is a mission scheduled for launch in December 2011 with anticipated first release of an intermediate catalogue some years after. Who are going to use these data? What other data should be obtained already now? These are issues which can be addressed together with the wider scientific community. GST sees its role in this community building and enabling process and is at the moment of this proceedings being written taking concrete steps toward the community by probing their interest to join the effort under the GREAT concept.

\section{References}

Bailer-Jones, C. 2008, these proceedings, p. 475 


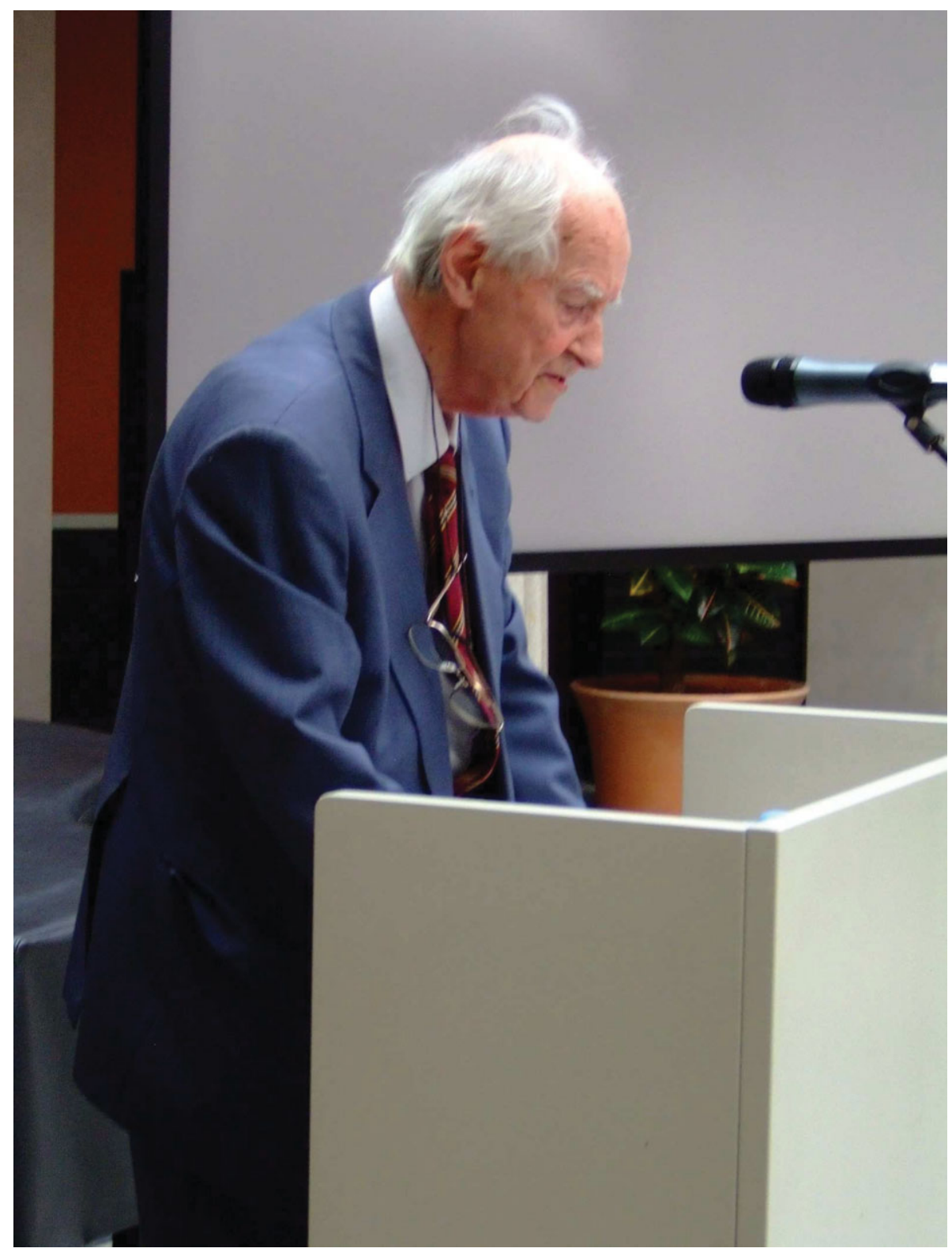

From Kapteyn to Gaia: Adriaan Blaauw reviewing 70 years of Galactic research, including half a century with Bengt Strömgren. 